\title{
Eddy viscosity for resolvent-based jet noise models
}

\author{
Ethan Pickering*, Georgios Rigas, Tim Colonius \\ California Institute of Technology, Pasadena, CA, USA \\ Denis Sipp \\ ONERA - The French Aerospace Lab, 92190 Meudon, France \\ Oliver T. Schmidt \\ University of California, San Diego, La Jolla, CA, USA
}

\begin{abstract}
Response modes computed via linear resolvent analysis have shown promising results for qualitatively modeling both the hydrodynamic and acoustic fields in jets when compared to data-deduced modes from high-fidelity, large-eddy simulations (LES). For an improved quantitative prediction of the near- and far-field, the role of Reynolds stresses must also be considered. In this study, we propose a methodology to deduce an eddy-viscosity model that optimally captures the nonlinear forcing of resolvent modes. The methodology is based on the maximization of the projection between resolvent analysis and spectral proper orthogonal decomposition (SPOD) modes using a Lagrangian optimization framework. For a Mach 0.4 round, isothermal, turbulent jet, four methods are used to increase the projection coefficients: linear damping, spatially constant eddy-viscosity field, a turbulent kinetic energy derived viscosity field, and an optimized eddy-viscosity field. The resulting projection coefficients for the optimized eddy-viscosity field between SPOD and resolvent can be increased to over $\mathbf{9 0 \%}$ for frequencies in the range $S t=0.35-1$ with significant improvements to $S t<0.35$. We find that the use of a frequency-independent turbulent kinetic energy turbulent viscosity model produces modes closely inline with optimal results, providing a preliminary eddy-viscosity resolvent model for jets.
\end{abstract}

\section{Introduction}

Commercial aircraft have seen reduced noise levels and increased fuel efficiency by increasing the bypass ratio of turbofan jet engines. However, the potential for additional noise reduction in modern aircraft is limited as larger engines with even higher bypass ratios would necessitate a complete redesign. Unlike commercial aircraft, military aircraft have not made significant progress in reductions to jet noise as they must maintain much more stringent performance metrics and thus have been unable to incorporate turbofans into their designs. The only viable pathway to reduce noise in these aircraft is through a more detailed understanding of noise generated by high speed turbulent flow. Therefore, an improved understanding of the fundamentals of noise mechanisms is necessary to inform further jet noise reduction techniques. The present work seeks this understanding through the development of robust, reduced-order jet noise models.

The principle tool for jet noise modeling is the acoustic analogy ${ }^{1}$ or one of its many generalizations. The analogy takes the form of a linear operator acting on fluctuations driven by a source term. In its most general form the operator ${ }^{2}$ represents the full linearized equations of momentum (Navier-Stokes), continuity, and energy, and the right-hand-side represents a forcing term that must be modeled in terms of partially known (experimentally or numerically) statistics of turbulence. Thus our starting point is the full compressible Navier-Stokes equations (plus continuity and energy equations), written compactly as:

$$
\frac{\partial q}{\partial t}=\mathcal{F}(q)
$$

where, $q$ is a vector of dependent variables. Inserting the standard Reynolds' decomposition about the mean flow:

$$
q(x, r, \theta, t)=\bar{q}(x, r)+q^{\prime}(x, r, \theta, t)
$$

*pickering@caltech.edu 
we can rewrite the full equations by segregating terms that are linear and nonlinear in the fluctuations to the LHS and RHS respectively. This gives

$$
\frac{\partial q^{\prime}}{\partial t}-\mathcal{A}(\bar{q}) q^{\prime}=f(x, r, \theta, t)
$$

with

$$
\mathcal{A}(\bar{q})=\frac{\partial \mathcal{F}}{\partial q}(\bar{q}) .
$$

Like Goldstein's analogy, this linear equation has a much richer Green's function than second- or third-order wave equations. Indeed, the operator accounts for both hydrodynamic (turbulent) fluctuations about the mean as well as the near- and far- field acoustics.

Inclusion of the hydrodynamic component in the propagator, seen as a curse by some, is more properly viewed as an opportunity because, beginning with the early experiments of Mollo-Christensen, ${ }^{3}$ it has become increasingly apparent that coherent structures in the near-field are responsible for the far-field radiated sound. ${ }^{4}$ These coherent structures take the spatio-temporal form of wavepackets, and have been found to be a significant source for aft angle noise ${ }^{4}$ and have also been found partially responsible for sideline noise. ${ }^{5,6}$ Such wavepackets were originally associated with instabilities of the mean turbulent flow, ${ }^{7-10}$ but it is increasingly apparent that they are properly viewed as a kind of "high-gain" forced response whereby the more random turbulent fluctuations are amplified through linear mechanisms.

Returning to equation (3), only a very limited set of Green's functions have been found numerically (see, for example $\left.{ }^{11-15}\right)$. Considering that a full characterization of the Green's function implies solving the full linearized equations of motion for a point source in any variable at any location within the jet, it is doubtful that this approach can fully succeed without further simplifications.

The important simplifying idea is to seek a low-rank approximation of the full Green's function. This can be achieved through a process called resolvent analysis. ${ }^{16-18}$ This analysis uses the Singular Value Decomposition (SVD) to decompose the LHS linear operator, which produces sets of mutually orthogonal input and response modes, which are ranked in terms of the corresponding gain between the input and response. By retaining only the highest-gain modes, a reduced-rank Green's function can be constructed.

This kind of linear analysis has been understood for many decades, but the computational power and algorithms to compute the resolvent decomposition for turbulent mean flows of interest have only come online recently. ${ }^{6,18-23}$ The existence of a low rank response has been verified in detail for round, turbulent jets, ${ }^{21}$ and there is an encouraging level of agreement between the high-gain response modes and and structures deduced from high-fidelity experimentally-verified large-eddy simulations (LES) of jets ${ }^{24,25}$ using a procedure called spectral proper orthogonal decomposition ${ }^{23}$ (SPOD).

The jet mechanisms uncovered through the previously mentioned resolvent analysis and SPOD comparisons of Schmidt et al. ${ }^{21}$ provide the basic analysis for which this paper seeks to extend and improve. The resolvent analysis of a round turbulent Mach $=0.4$ jet across Strouhal numbers $S t=[0.2-2]$ presents response modes of two mechanisms, Kelvin-Helmholtz-type (KH) and Orr-type. The KH-type mode emerges as the dominant high-gain mode from $S t=0.3-1.8$, while the Orr-type modes dominate both at lower and higher frequencies, as well as in the suboptimal resolvent modes. From their analysis the KH-type modes provided significant qualitative agreement with corresponding SPOD modes, while Orr-type modes both optimal and suboptimal showed significant discrepancies in both structure and spatial locations. Although the Mach 0.4 jet resolvent analysis presents a low-rank response for a large band of frequencies, the Orr-type modes account for a substantial amount of flow energy and must be adequately captured.

Therefore, to constitute a complete jet noise model it is still required to pose a model for the statistics of a sufficient set of input modes (i.e. suboptimal modes). Theoretically, it can be shown that if the inputs are white noise (spatially uncorrelated), then the resolvent response modes must be identical to the SPOD modes. ${ }^{23}$ Discrepancies between SPOD modes and resolvent response therefore implies correlation between the input modes. As noted above, the agreement between SPOD (true response) and resolvent modes (theoretical response) is only qualitative; quantitative discrepancies originate from the presence of correlation between the resolvent inputs. Such correlation can be represented as a colored noise process, and some approaches to determine its covariance structure based on matching (partially) observed statistics have been developed. ${ }^{26,27}$

Here we examine the extent to which the addition of an eddy-viscosity model in the resolvent framework can further reduce associated correlation between the resolvent input modes, such that white-noise (spatially) 
models for their coefficients would suffice. The eddy viscosity is a well-established approach insofar as the mean flow is concerned, but its action on the fluctuation field is less clear. In resolvent jet computations to date, ${ }^{6,18,19,21}$ the Reynolds number has typically been chosen to be as high as possible, but the resolvent computations becomes intractable when the molecular viscosity alone is used-the sub-optimal input and output modes then obtain a structure that is too fine to resolve even on a mesh as fine as the LES-thus necessitating a compromise.

The use of eddy-viscosity models, i.e., turbulence models, have been used in various mean flow stability analyses to account for additional mixing introduced by fine-scale turbulence. One of the first global stability approaches, Crouch et al., ${ }^{28}$ computed global responses for buffet modes through the Reynolds Averaged Navier-Stokes equations closed via a Spalart-Allmaras turbulence model. Oberleithner et al. ${ }^{29}$ and Rukes et al. ${ }^{30}$ significantly improved global linear stability results through the employment of a Boussinesq approximation and anisotropic eddy-viscosity model, respectively, for strongly swirling jets. Other studies on eddy-viscosity models include Sartor et al. ${ }^{31}$ in stabilizing buffet modes, Tammisola \& Juniper ${ }^{32}$ on global stability analysis for fuel injectors, where they found that the choice of the model for turbulent dissipation is less crucial than the choice of including it in improving global mode shapes, and Mettot et al. ${ }^{33}$ where the use of an eddy-viscosity model was implemented for improved frequency control maps.

Recently Morra et al. ${ }^{34}$ also studied the relevance of eddy viscosity for resolvent analysis for wall bounded flows. They found that the addition of a prescribed eddy-viscosity model for wall bounded flows in the resolvent analysis lead to significant improvements in predicting the measured spatio-temporal power spectral densities. However, they also conclude that contrary to wall-bounded flows, jets have a weak dependence on a turbulent eddy viscosity, here we show the contrary for turbulent jets.

In this study, we develop an optimization framework to identify an eddy-viscosity field that leads to the closest alignment possible between the SPOD and resolvent modes for a round, turbulent Mach 0.4 jet at azimuthal wavenumber $m=0$ (other azimuthal modes are relegated to future work). In the next section we outline the governing equations, resolvent analysis, SPOD, and the optimization framework developed to alignment the optimal modes. We propose four methods for improving the alignment between the modes and present each method's results. We conclude by adopting a frequency-independent turbulent kinetic energy (TKE) eddy-viscosity model and demonstrate its performance over all Strouhal numbers considered.

\section{Methods}

\section{A. Governing equations}

For the round isothermal jet considered in this paper, the governing equations are cast in the cylindrical coordinate frame with variable state vector $q=\left[\rho^{\prime}, u_{x}^{\prime}, u_{r}^{\prime}, u_{\theta}^{\prime}, T^{\prime}\right]$. Equations are non-dimensionalized by centerline nozzle exit values, with subscript $j$. Mach number and Reynolds number are defined as $M_{j}=U_{j} / a_{j}$ and $R e_{j}=\frac{\rho_{j} U_{j} D}{\mu_{j}}$, respectively, with isothermal conditions $T_{j} / T_{\infty}=1$, where $U$ jet centerline axial velocity, $a$ speed of sound, $\rho$ density, $D$ nozzle diameter, $\mu$ dynamic viscosity, $T$ temperature, and $\infty$ free-stream conditions. Frequency is given by the dimensionless Strouhal number $S t=\omega /\left(2 \pi M_{j}\right)$ where $\omega$ is the angular frequency. The LES database consists of $10^{4}$ snapshots, separated in time by $\Delta t c_{\infty} / D=0.2$ with $R e_{j}=4.5 \times 10^{5}$. Snapshots are read from an interpolated grid of dimension $656 \times 138 \times 128$ spanning the domain $x / D, r / D, \theta \in[0,30] \times[0,6] \times[0,2 \pi]$ where $[x / D, r / D]=[0,0]$ denotes the centerline of the nozzle exit.

For the round, statistically-stationary, turbulent, Mach 0.4 jet considered in this manuscript, the compressible Navier-Stokes, energy, and continuity equations are linearized via a standard Reynolds decomposition, and Fourier transformed both temporally and azimuthally to the compact expression

$$
\left(i \omega \mathbf{I}-\mathbf{A}_{m}\right) \boldsymbol{q}_{m, \omega}=\boldsymbol{L}_{m, \omega} \boldsymbol{q}_{m, \omega}=\boldsymbol{f}_{m, \omega} .
$$

Where $\boldsymbol{L}_{m, \omega}$ is the forward linear operator, $\boldsymbol{q}=\left[\rho^{\prime}, u_{x}^{\prime}, u_{r}^{\prime}, u_{\theta}^{\prime}, T^{\prime}\right]$ is the state vector, $\boldsymbol{f}$ constitutes the nonlinear forcing in each variable, $\omega$ is the frequency, and $m$ is the azimuthal mode number. Discretization is performed on the streamwise plane with fourth-order summation by parts finite differences from Mattsson and Nordström ${ }^{35}$ and the polar singularity is treated as in Mohseni and Colonius. ${ }^{36}$ Additionally, nonreflecting boundary conditions are implemented at the domain boundaries. 


\section{B. Resolvent analysis}

We can rewrite equation 5 by defining the resolvent operator, $\boldsymbol{R}_{\omega, m}=\boldsymbol{L}_{m, \omega}^{-1}$,

$$
\boldsymbol{q}_{m, \omega}=\boldsymbol{R}_{m, \omega} \boldsymbol{f}_{m, \omega} .
$$

Introducing the compressible energy norm, ${ }^{37}$

$$
\left\langle\boldsymbol{q}_{1}, \boldsymbol{q}_{2}\right\rangle_{E}=\iiint \boldsymbol{q}_{1}^{*} \operatorname{diag}\left(\frac{\bar{T}}{\gamma \bar{\rho} M^{2}}, \bar{\rho}, \bar{\rho}, \bar{\rho}, \frac{\bar{\rho}}{\gamma(\gamma-1) \bar{T} M^{2}}\right) \boldsymbol{q}_{2} r d x d r d \theta=\boldsymbol{q}_{1}^{H} \boldsymbol{W} \boldsymbol{q}_{2},
$$

(where superscript $H$ denotes the Hermitian transpose) via the matrix $\boldsymbol{W}$ to the forcing and response gives the weighted resolvent operator

$$
\boldsymbol{H}_{m, \omega}=\boldsymbol{W}_{q}^{\frac{1}{2}}\left(i \omega \mathbf{I}-\mathbf{A}_{m}\right)^{-1} \boldsymbol{W}_{f}^{-\frac{1}{2}} .
$$

Taking the singular value decomposition of the modified resolvent operator gives

$$
\boldsymbol{H}_{m, \omega}=\boldsymbol{Q}_{m, \omega} \boldsymbol{\Sigma} \boldsymbol{F}_{m, \omega}^{H}
$$

where $\boldsymbol{Q}_{m, \omega}=\left[q_{m, \omega}^{1}, q_{m, \omega}^{2}, \ldots, q_{m, \omega}^{N}\right]$ are the optimal response modes, $\boldsymbol{F}_{m, \omega}=\left[f_{m, \omega}^{1}, f_{m, \omega}^{2}, \ldots, f_{m, \omega}^{N}\right]$ are the optimal forcing modes, and $\boldsymbol{\Sigma}=\left(\sigma_{1}, \sigma_{2}, \ldots, \sigma_{N}\right)$ are the optimal gains. Accounting for the numerical quadrature weighting matrix normalizes each forcing and response mode such that they are orthonormal and may be compared to their corresponding SPOD modes.

\section{Spectral Proper Orthogonal Decomposition}

Spectral proper orthogonal decomposition, ${ }^{38,39}$ finds an optimal set of orthogonal modes that describe a dataset, but unlike spatial-only POD, produces modes that express both spatial and temporal correlation in the data. Like DMD, each mode is associated with a unique frequency, but are naturally ranked by energy and, through appropriate averaging, account for statistical variability in turbulent flows. ${ }^{23}$

Decomposing the LES database, $\mathcal{Q}$, in the azimuthal and temporal dimensions via the discrete Fourier transform gives the decomposed data matrices, $\mathcal{Q}_{m, \omega}$. The cross-spectral density tensor at a given frequency wavenumber pair is then given by

$$
\mathbf{S}_{m, \omega}=\mathcal{Q}_{m, \omega} \mathcal{Q}_{m, \omega}^{H}
$$

and the SPOD eigenvalue problem presented by Lumley ${ }^{38,39}$ can be solved

$$
\mathbf{S}_{m, \omega} \mathbf{W} \boldsymbol{Q}_{s, m, \omega}=\boldsymbol{Q}_{s, m, \omega} \boldsymbol{\Lambda}_{m, \omega} .
$$

The SPOD modes are represented by the columns of $\boldsymbol{Q}_{s, m, \omega}$ (subscript $s$ denoting SPOD response mode matrix) and are ranked by the diagonal matrix of eigenvalues $\boldsymbol{\Lambda}_{m, \omega}$. The modes are orthonormal in the Chu energy norm, $\boldsymbol{W}$, and satisfy $\boldsymbol{Q}_{s, m, \omega}^{*} \boldsymbol{W} \boldsymbol{Q}_{s, m, \omega}=\boldsymbol{I}$. The cross-spectral density tensor may also be expanded as $\mathbf{S}_{m, \omega}=\boldsymbol{Q}_{s, m, \omega} \boldsymbol{\Lambda}_{m, \omega} \boldsymbol{Q}_{s, m, \omega}^{H}$.

Recently, multiple authors have reported the theoretical connection between the resolvent analysis and SPOD. ${ }^{18,21,23,40}$ These analyses are connected through their response and forcing cross-spectral density (CSD) matrices by the resolvent operator. Assuming any pair of wavenumbers/frequencies, we drop the $m$ and $\boldsymbol{\omega}$ subscripts from the $\boldsymbol{S}$ and define the response CSD for response $\boldsymbol{S}_{q q}=\left\langle q q^{H}\right\rangle$ and $\boldsymbol{S}_{f f}=\left\langle f f^{H}\right\rangle$ for forcing, with $\langle\cdot\rangle$ as the expectation operator. Applying the expectation operator to equation 6 multiplied by its complex conjugate gives,

$$
\boldsymbol{S}_{q q}=\left\langle q q^{H}\right\rangle=\left\langle\boldsymbol{R} f f^{H} \boldsymbol{R}^{H}\right\rangle=\boldsymbol{R} \boldsymbol{S}_{f f} \boldsymbol{R}^{H} .
$$

The above equation directly relates the cross-spectral densities of forcing and response terms with the resolvent operator. Furthermore, if the forcing CSD is spatially white, meaning $S_{f f}=\alpha(\omega) \boldsymbol{I}$, where $\alpha(\omega)$ is a scalar frequency spectrum, then equation 12 reduces to:

$$
\begin{aligned}
& \boldsymbol{S}_{q q}=\boldsymbol{R} \alpha \boldsymbol{R}^{H}=\boldsymbol{Q} \boldsymbol{\Sigma} \boldsymbol{F}^{H} \alpha \boldsymbol{F} \boldsymbol{\Sigma} \boldsymbol{Q}^{H}=\boldsymbol{Q} \alpha \boldsymbol{\Sigma}^{2} \boldsymbol{Q}^{H} \\
& \boldsymbol{S}_{q q}=\boldsymbol{Q}_{s} \boldsymbol{\Lambda} \boldsymbol{Q}_{s}^{H}
\end{aligned}
$$


Therefore, the resolvent modes will be identical to the set of SPOD modes under spatially-white forcing.

Real turbulent flows, of course, are not white. ${ }^{23,26}$ To assess the degree of coloring between the resolvent and SPOD analyses, projections can be made between the SPOD output, $\boldsymbol{Q}_{\boldsymbol{s}}$, and the resolvent output, $\boldsymbol{Q}$. This connection has been explored within the jet community in both the near-field ${ }^{21,23}$ and the far-field. ${ }^{18,41}$

This paper seeks to find a (linear) turbulence model such that when it is included in the linear operator $\boldsymbol{L}$, the altered resolvent modes are best aligned with the observed SPOD modes. If a perfect alignment could be achieved, then a spatially white-noise forcing could be applied to the (modified) linear operator to produce the observed spectrum of large-scale turbulent structures (and ultimately their radiated sound). In the next section we present an optimization framework designed to achieve this goal.

\section{Optimization Framework}

An optimization is constructed with the goal of discerning an optimal modification to the linear operator such that the projection coefficients between SPOD and resolvent modes, are maximized. To do so we prescribe a constrained optimization problem subject to the governing equations, the resolvent analysis, a normalization constraint, and constraints associated with an assumed ansatz for modifying the operator. For the latter we consider linear damping and eddy-viscosity frameworks. All components of this optimization are coupled as a Lagrangian functional and the variations taken with respect to each parameter allow for the identification of the functional's stationary point, or maximum.

To construct the Lagrangian functional we return to the forward equation 5 and substitute $\boldsymbol{L}$ with $\boldsymbol{L}_{\chi}$, the modified operator which introduces the turbulence models discussed in the following section. We then multiply both sides $\boldsymbol{L}_{\chi}^{H}$ which emits the inverse resolvent eigenvalue problem, $\boldsymbol{L}_{\chi}^{H} \boldsymbol{L}_{\chi}=\boldsymbol{\sigma}^{-2}$ :

$$
\boldsymbol{q}=\boldsymbol{\sigma}^{2} \boldsymbol{L}_{\chi}^{H} \hat{\boldsymbol{f}}
$$

We then enforce an energy constraint of the output through the Chu norm

$$
\hat{\boldsymbol{q}}^{H} \boldsymbol{W} \hat{\boldsymbol{q}}=\mathbf{1}
$$

Then the cost function is constructed as

$$
\mathcal{J}=\hat{\boldsymbol{q}}^{H} \boldsymbol{W} \hat{\boldsymbol{q}}_{\boldsymbol{s}} \hat{\boldsymbol{q}}^{\mathrm{s}}{ }^{H} \boldsymbol{W} \hat{\boldsymbol{q}}
$$

which seeks to maximize the square of the projection between the optimal SPOD mode, $\boldsymbol{q}_{\boldsymbol{s}}$, and first resolvent mode, $\boldsymbol{q}$. By taking the square of the projection, $\boldsymbol{q}^{H} \boldsymbol{W} \boldsymbol{q}_{\boldsymbol{s}}$, we ensure that the cost is real. For brevity, $\boldsymbol{Q}_{\boldsymbol{s}}=\hat{\boldsymbol{q}}_{s} \hat{\boldsymbol{q}}_{s}{ }^{H}=\boldsymbol{Q}_{s}^{H}$.

We now construct the full Lagrangian functional combining the cost function 17, forward equation 5, resolvent eigenvalue problem 15 , and the constraint 16 to give

$$
\mathcal{L}=\hat{\boldsymbol{q}}^{H} \boldsymbol{W} \boldsymbol{Q}_{\boldsymbol{s}} \boldsymbol{W} \hat{\boldsymbol{q}}-\tilde{\boldsymbol{q}}^{H} \boldsymbol{W}\left(\boldsymbol{L}_{\chi} \hat{\boldsymbol{q}}-\hat{\boldsymbol{f}}\right)-\tilde{\boldsymbol{f}}^{H} \boldsymbol{W}\left(\hat{\boldsymbol{q}}-\sigma^{2} \boldsymbol{L}_{\chi}^{H} \hat{\boldsymbol{f}}\right)-\lambda\left(\hat{\boldsymbol{q}}^{H} \boldsymbol{W} \hat{\boldsymbol{q}}-\mathbf{1}\right)+c . c .
$$

The newly acquired terms, $(\tilde{\boldsymbol{q}}, \tilde{\boldsymbol{f}}, \lambda)$, in the Lagrangian functional are Lagrangian multipliers and c.c. are the complex conjugates to ensure real values. This results in a functional dependent on the six variables:

$$
\mathcal{L}([\boldsymbol{q}, \boldsymbol{f}, \sigma],[\tilde{\boldsymbol{q}}, \tilde{\boldsymbol{f}}, \lambda]) .
$$

To find the maximum Lagrangian functional, the variations with respect to all variables must be considered. Taking variations with respect to each multiplier gives:

$$
\frac{\partial \mathcal{L}}{\partial[\tilde{\boldsymbol{q}}, \tilde{\boldsymbol{f}}, \boldsymbol{\lambda}]}([\delta \tilde{\boldsymbol{q}}, \delta \tilde{\boldsymbol{f}}, \delta \lambda])=-\delta \tilde{\boldsymbol{q}}^{H} \boldsymbol{W}\left(\hat{\boldsymbol{L}_{\chi}} \boldsymbol{q}-\hat{\boldsymbol{f}}\right)-\delta \tilde{\boldsymbol{f}}^{H} \boldsymbol{W}\left(\hat{\boldsymbol{q}}-\sigma^{2} \boldsymbol{L}_{\chi}^{H} \hat{\boldsymbol{f}}\right)-\delta \lambda\left(\hat{\boldsymbol{q}}^{H} \boldsymbol{W} \hat{\boldsymbol{q}}-\mathbf{1}\right)+c . c .
$$

It is easily seen from our governing equations that $\boldsymbol{L}_{\chi}^{\hat{q}}=\hat{\boldsymbol{f}}, \hat{\boldsymbol{q}}=\sigma^{2} \boldsymbol{L}_{\chi}^{H} \hat{\boldsymbol{f}}$, and $\hat{\boldsymbol{q}}^{H} \boldsymbol{W} \hat{\boldsymbol{q}}=\mathbf{1}$, therefore, the stationary point with respect to the multipliers is automatically satisfied. Taking variations with respect to the three resolvent variables gives: 


$$
\begin{aligned}
& \frac{\partial \mathcal{L}}{\partial \hat{\boldsymbol{q}}}=\left(\boldsymbol{Q}_{\boldsymbol{s}} \boldsymbol{W} \hat{\boldsymbol{q}}-\boldsymbol{L}_{\chi}^{H} \tilde{\boldsymbol{q}}-\tilde{\boldsymbol{f}}-\lambda \hat{\boldsymbol{q}}\right) \delta \hat{\boldsymbol{q}} \quad=0 \\
& \frac{\partial \mathcal{L}}{\partial \hat{\boldsymbol{f}}}=\left(\tilde{\boldsymbol{q}}+\sigma^{2} \boldsymbol{L}_{\chi} \tilde{\boldsymbol{f}}\right) \delta \hat{\boldsymbol{f}} \quad=0
\end{aligned}
$$

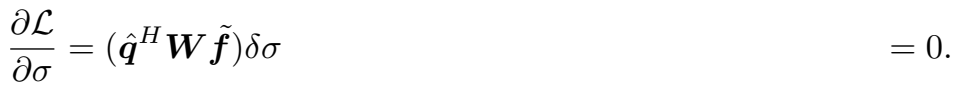

The stationary point is then met by constructing the following matrix and solving for the Lagrangian multipliers:

$$
\left[\begin{array}{ccc}
-\boldsymbol{L}_{\chi}^{H} & -\boldsymbol{I} & -\hat{\boldsymbol{q}} \\
\boldsymbol{I} & \boldsymbol{L}_{\chi} \sigma^{2} & 0 \\
0 & \hat{\boldsymbol{q}}^{H} \boldsymbol{W} & 0
\end{array}\right]\left[\begin{array}{c}
\tilde{\boldsymbol{q}} \\
\tilde{\boldsymbol{f}} \\
\lambda
\end{array}\right]=\left[\begin{array}{c}
-\boldsymbol{Q}_{\boldsymbol{s}} \boldsymbol{W}^{H} \hat{\boldsymbol{q}} \\
0 \\
0
\end{array}\right]
$$

To perform the optimization, a final variation is taken with respect to a prescribed parameter, or set of parameters, $\chi$, that affect the forward operator $\boldsymbol{L}_{\chi}$. The variation with respect to these parameters takes the form

$$
\frac{\partial \mathcal{L}}{\partial \chi}=-\tilde{\boldsymbol{q}}^{H} \boldsymbol{W} \frac{\partial \boldsymbol{L}_{\chi}}{\partial \chi} \hat{\boldsymbol{q}}+\tilde{\boldsymbol{f}}^{H} \boldsymbol{W} \sigma^{2} \frac{\partial \boldsymbol{L}_{\chi}^{H}}{\partial \chi} \hat{\boldsymbol{f}}+c . c=0 .
$$

The variation provides the direction of gradient ascent for the optimization parameter and the updated parameter is

$$
\chi_{k+1}=\chi_{k}+\alpha \frac{\partial \mathcal{L}}{\partial \chi}
$$

where $k$ is the iteration number and $\alpha$ is a step size determined through a root finding algorithm or a line search.

We do not know in general if the optimization problem here yields global or local maxima, and particularly when $\chi$ involves many degrees of freedom the topology of the cost function may be very complex. We briefly discuss the observed topology in connection with the specific models considered (and their resulting parameters $\chi$ ) in the next section.

\section{E. Turbulence models considered}

Four methods for including a turbulence model in the linear operator are investigated. The first model is simplest: a linear damping term is added to the model, whereas the other three models are based on eddy-viscosity concepts. The eddy viscosity differs from linear damping in that it acts on derivatives of the fluctuations to give a damping that is proportional to the wavenumber squared. The simplest eddy viscosity we test is a constant model, which is equivalent to specifying a constant, effective Reynolds number. The remaining two models consider spatially-varying eddy viscosity. The first of these is based on a simple closure based on turbulent kinetic energy (TKE) and a length scale, and the last model directly optimizes the entire spatial eddy-viscosity field under a constraint of positivity. For the first three models, where the form of the model is determined a priori, we still use optimization to determine the best possible overall constant.

\section{Linear damping term (finite-time-horizon resolvent)}

The first parameter studied within the optimization is that of a linear damping, which is equivalent to finite-time-horizon resolvent analysis introduced by Jovanović. ${ }^{42}$ A recent resolvent study by Yeh \& Taira ${ }^{43}$ used a finite-time approach to localize the forcing and responses modes of an airfoil. Without the addition of a finite-horizon, the forcing modes in their study extend infinitely in the upstream direction and in the downstream direction for response modes. However, in real flows there exist significant short term (i.e. finite-time) dynamics of interest, such as intermittent behavior and development times for flow structures. A similar argument can be made with jet flows, where wavepackets may not be adequately bounded in the axial 
direction when compared to their corresponding SPOD modes, which by construction accurately account for the intermittent behavior and development times of structures in real flows.

For this model, the operator is modified so that

$$
\boldsymbol{L}_{\chi}=\boldsymbol{L}-\beta \boldsymbol{I} .
$$

where $\beta=1 / \tau>0$, and $\tau$ is the desired temporal decay rate. We used the optimization framework above to select, as a function of frequency, the value of $\beta$ that best aligns the principle resolvent and SPOD modes. The optimization (at each frequency) is with respect to a single constant, that is $\chi=[\beta]$. For this optimization an initial condition of $\beta=0$ was used. The sensitivity of the optimal $\beta$ to this initial guess was checked for selected frequencies and verified that (at least for $\beta>0$ ), we achieved a global maximum.

\section{Constant eddy-viscosity field}

For the eddy-viscosity models, we modify the linear operator to include a Boussinesq-type eddy viscosity, that is we take

$$
\boldsymbol{L}_{\chi}=\boldsymbol{L}+\boldsymbol{L}_{T}\left(\mu_{T}\right)
$$

where $L_{T}$ is the discretized operator associated with the linearization of equations including the eddy-viscosity model. With a slight abuse of notation, we write

$$
\boldsymbol{L}_{T}=\left[\begin{array}{c}
\boldsymbol{L}_{T, \text { Cont. }} \\
\boldsymbol{L}_{T, \text { Mom. }} \\
\boldsymbol{L}_{T, \text { Energy }}
\end{array}\right]=\operatorname{discretization~of}\left[\begin{array}{c}
0 \\
\nabla \cdot\left[\mu_{T}\left((\nabla \boldsymbol{u})+(\nabla \boldsymbol{u})^{T}+\lambda \Theta \mathbb{I}\right)\right] \\
\gamma M^{2} \mu_{T}\left[\frac{1}{2}\left\{(\nabla \boldsymbol{u})+(\nabla \boldsymbol{u})^{T}\right\}:\left\{(\nabla \boldsymbol{u})+(\nabla \boldsymbol{u})^{T}\right\}+\lambda \Theta^{2}\right]
\end{array}\right]
$$

where $\Theta=\nabla \cdot \boldsymbol{u}$ is the dilatation, $\mathbb{I}$ is the second order identity tensor, and $\lambda=-2 / 3$. The eddy-viscosity term, $\mu_{T}$, is nondimensionalized by $\rho_{j}, U_{j}, D$ and in sum with the molecular viscosity presents an effective viscosity $\mu_{e f f}=\mu_{T}+1 / R e_{j}$. However, in the eddy-viscosity models presented here, $\mu_{T}$ tends to be much larger than $1 / R e_{j}$ and as such $\mu_{e f f} \approx \mu_{T}$.

The first eddy-viscosity model we consider is a constant field, $\mu_{T}=\frac{1}{R e_{T}}$, with constant turbulent Reynolds number. The optimization (again at each frequency) is thus over a single parameter, $\chi=\left[\operatorname{Re}_{T}\right]$. For this case, an initial condition of $R e_{T}=3 \times 10^{4}$ was used. Again, the sensitivity of the optimal $R e_{T}$ to the initial guess was checked for selected frequencies and verified to achieve a global maximum.

\section{Turbulent kinetic energy eddy-viscosity model}

A large variety of spatially varying eddy-viscosity models have been proposed for the mean Reynolds stresses. As a first step towards a more comprehensive evaluation, we consider a specific model based on turbulent kinetic energy (TKE). ${ }^{44}$ This model was primarily chosen due to its simplicity and availability of the corresponding quantities from the LES database, though as we will show it is surprisingly effective. The model takes the form

$$
\mu_{T, T K E}=\bar{\rho} c k^{1 / 2} l_{m}
$$

where $c$ is a scaling constant, $k$ is the mean flow turbulent kinetic energy, and $l_{m}$ is a chosen length scale representative of the mean shear layer thickness. We choose this constant as the width of the shear layer where the turbulent kinetic energy is more than $10 \%$ of its maximum value, at each axial location. The resulting eddy-viscosity field (relative to c) is plotted in figure 1 , and the black lines denote the location where TKE is $10 \%$ of its maximum value. Note that after $\approx x / D=5, l_{m}$ is defined from $r / D=0$ to the $10 \%$ TKE contour. For this model, the single optimization parameter (again at each frequency) is the overall constant, and we take $\chi=[c]$. This optimization was initialized with $c=0.01$ and similar to the previous methods, the sensitivity to this initial guess was checked and achievement of a global maximum met. 


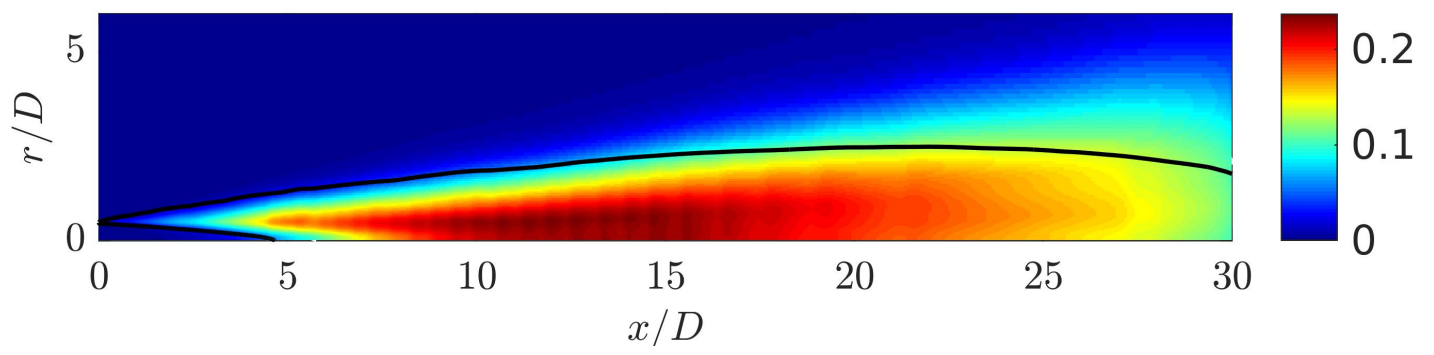

Figure 1. Computed eddy-viscosity field via the turbulent kinetic energy model, $\mu_{T, T K E}=\bar{\rho} c k^{1 / 2} l_{m}$, with $c=1$. The black lines denote the region of $>10 \%$ maximum TKE which defines the length scale, $l_{m}$.

\section{Optimal eddy-viscosity field}

The final method consists of optimizing for the entire spatial eddy-viscosity field. The purpose of such an optimization is not for creation of an eddy-viscosity model, but to understand what might be the upper bound (in terms of the optimization) of any such model. Here we take, simply $\chi=\left[\mu_{T}\right]$, so that the number of optimization parameters is now much larger and equal to the number of grid points in the $x-r$ plane. For this case, a complete assessment of the sensitivity of initial conditions and proof of a global maximum are intractable due to its complexity, and reserve future efforts to a robust understanding of the optimization topology. Considering the previous methods' insensitivity to initial conditions, ability to attain the global maximum, and the optimization upper bound of $\boldsymbol{q}_{s} \boldsymbol{W} \boldsymbol{q}=1$, we progress with the optimal eddy-viscosity field optimization confident the results are at or near the true global maximum. Important for this confidence is a well-chosen initial condition. To this end, the initial condition provided for the full-field optimization is that of the optimal $R_{T}$ constant eddy viscosity result.

\section{Results}

\section{A. Optimal mode alignment}

We first present the resulting mode shapes of the entire state vector, $\boldsymbol{q}^{\prime}=\left[\rho^{\prime}, u_{x}^{\prime}, u_{r}^{\prime}, u_{\theta}^{\prime}, T^{\prime}\right]$, and pressure ( $p^{\prime}$ found by equation of state $p=\rho T / \gamma M^{2}$ ) for the optimal viscosity case. Comparisons are made between the optimal result with SPOD and the previously reported results of Schmidt et al., ${ }^{21}$ where a constant effective eddy viscosity, $R e_{T}=3 \times 10^{4}$ was used (resulting in a resolvent analysis conducted at a Reynolds number $R e_{j} / R e_{T}=15$ times lower than the true jet Reynolds number). Pressure mode shapes and their corresponding optimal projection coefficients are then shown for each method considered, $R e_{T}=3 \times 10^{4}$, linear damping, constant eddy-viscosity field, TKE eddy-viscosity model, and the optimal eddy-viscosity field. All results presented here are for the axisymmetric mode $m=0$, and we consider a range of frequencies from $S t=0.05$ to 1 .

Figure 2 displays the real of the fluctuating field for all state variables including pressure from SPOD, optimal viscosity, and $R e_{T}=3 \times 10^{4}$ at $S t=0.05$. Contours are given by $0.5\left\|\cdot^{\prime}\right\|_{\infty}$ of the SPOD mode, where $\cdot^{\prime}$ is the fluctuating variable in question (with $\left\|\cdot^{\prime}\right\|_{\infty}$ values: $\left[\rho^{\prime}, u_{x}^{\prime}, u_{r}^{\prime}, u_{\theta}^{\prime}, T^{\prime}, p^{\prime}\right]=[2.8,198.6,46.0,37.2,1.2,10.4] \times$ $10^{-3}$ for this case), with red and blue as positive and negative values respectively. This color scheme, based upon each SPOD mode, is maintained for the remainder of the contoured figures. It is immediately apparent that the optimal viscosity method is able to closely match the mode shapes of SPOD for all variables, with the exception of $u_{\theta}^{\prime}$.

In fact, the discrepancy between $u_{\theta}^{\prime}$ terms highlight some of the statistical errors contained within SPOD modes. For the axisymmetric case, $m=0$, we expect $u_{\theta}^{\prime}$ to be quite small for spatially-varying eddy-viscosity fields and identically to zero with a constant eddy-viscosity field. The SPOD mode, in contrast to $u_{\theta}^{\prime}$ quite small, provides a rather incoherent $u_{\theta}^{\prime}$ result with velocity only 5 times smaller than $u_{x}^{\prime}$. This corresponds to a $u_{\theta}^{\prime}$ contribution in the projection coefficient $\boldsymbol{q}_{s} \boldsymbol{W} \boldsymbol{q}$ of $\approx 0.08$, bounding the physical maximum of the optimization to $\boldsymbol{q}_{s} \boldsymbol{W} \boldsymbol{q}=0.92$ without considering additional error in the other variables. These statisti- 

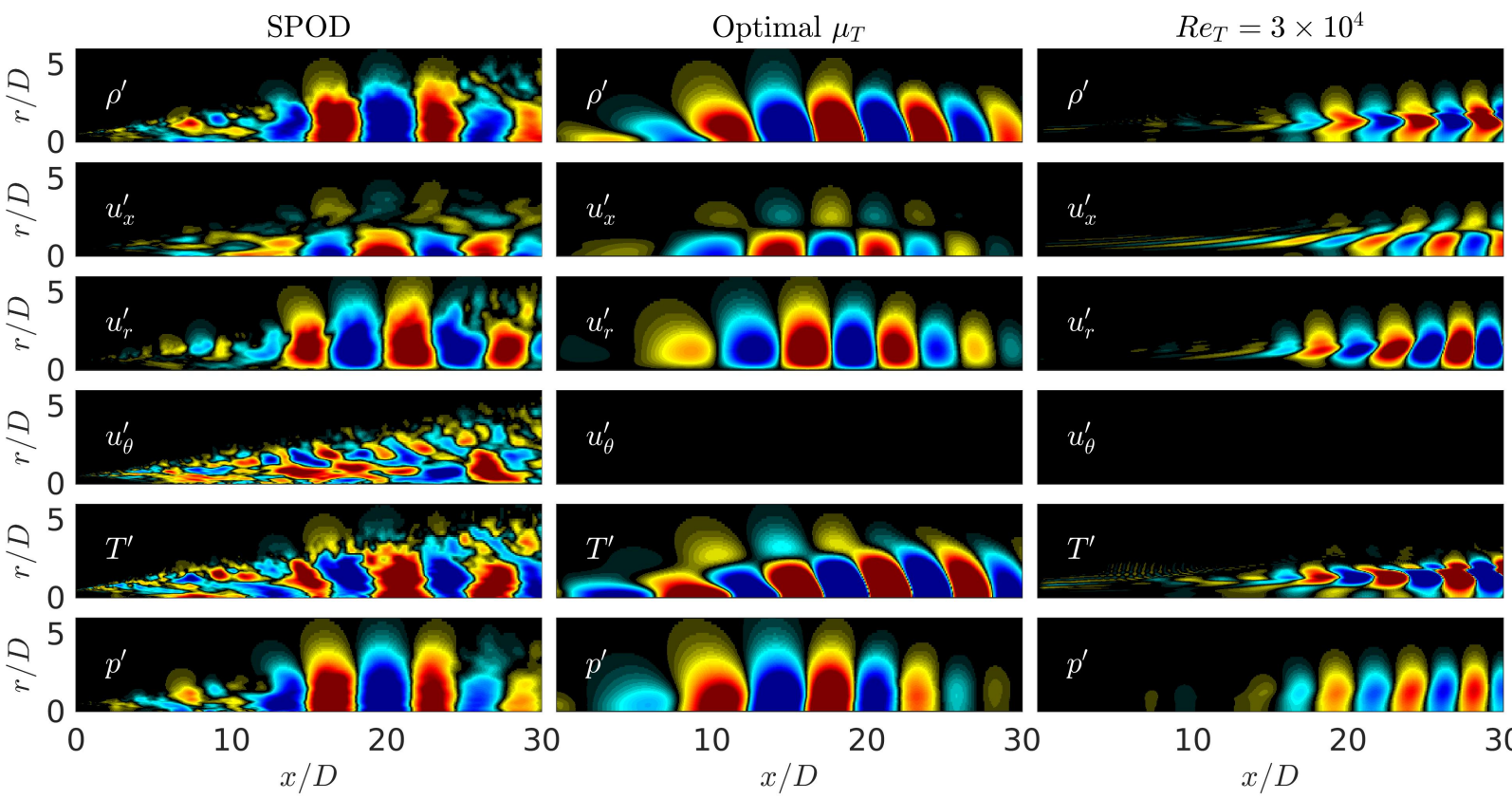

Figure 2. Real component of the fluctuating response state variables, $\boldsymbol{q}^{\prime}=\left[\rho^{\prime}, u_{x}^{\prime}, u_{r}^{\prime}, u_{\theta}^{\prime}, T^{\prime}\right]$, and pressure, $p^{\prime}$, at $S t=0.05$. The columns display SPOD, optimal eddy viscosity, and $R_{T}=3 \times 10^{4}$ modes from left to right respectively. Contours are given by $0.5\left\|\cdot \cdot^{\prime}\right\|_{\infty}$ of the SPOD mode, where $\cdot^{\prime}$ is the fluctuating variable in question (with $\left\|\cdot^{\prime}\right\|_{\infty}$ values: $\left[\rho^{\prime}, u_{x}^{\prime}, u_{r}^{\prime}, u_{\theta}^{\prime}, T^{\prime}, p^{\prime}\right]=[2.8,198.6,46.0,37.2,1.2,10.4] \times 10^{-3}$ ), with red and blue as positive and negative values respectively.

cal errors are linked to the weak low-rank behavior associated with Orr-type modes seen at low and high frequencies and within the suboptimal modes when compared to the stronger, low-rank behavior associated with KH-type modes at moderate frequencies (i.e. $S t=[0.3-0.8]) .{ }^{21}$ This leads us to expect optimization results of the Orr-type modes with slightly lower projection coefficient agreement due to SPOD statistical limitations. Therefore, we proceed cognizant of the limitations for Orr-type mode alignment, and the optimization method's potential for non-physical alignment with SPOD, but remain confident in the method given the optimization did not align with the statistical discrepancies of $u_{\theta}^{\prime}$ in figure 2 .

We also note that the pressure field, as a quantity of particular interest for jet noise, provides a rather representative mode shape for each case. As such, we proceed by visualizing only the real fluctuating pressure component for the remainder of the study, however the projection coefficients, $\left|\boldsymbol{q}_{s} \boldsymbol{W} \boldsymbol{q}\right|$, maintain and present the full state results.

Figure 3 shows the pressure modes and reports the alignment coefficients at the lowest frequencies, $S t=0.05$ and 0.2. The top row shows the dominant SPOD mode from the LES, the second row shows the dominant resolvent mode with fixed $\operatorname{Re}_{T}=3 \times 10^{4}$, and the remaining rows show the 4 optimized eddyviscosity models. At low frequency, the resolvent analysis at $R e_{T}=3 \times 10^{4}$ fails to capture the low frequency mode shapes. The optimized models, by contrast, have much better alignment with SPOD, increasing the projection coefficient by as much as 7-fold, and displaying a wavepacket structure consistent with the SPOD mode.

As expected, the optimal eddy viscosity field reached the highest alignment between the methods. It is followed by optimal constant eddy viscosity, the optimal TKE eddy-viscosity model, and optimal linear damping. We can see that although the linear damping is able to increase the projection coefficient, the performance is significantly inferior to the eddy-viscosity models, likely due to its monolithic damping effect over all wavenumbers. With one exception (the TKE model at $S t=0.05$ ), all the eddy-viscosity models perform similarly, reaching projection coefficients of about $70 \%$.

At these low frequencies, $S t<0.3$, the non-optimized resolvent spectrum for a Mach 0.4 jet is dominated by Orr-type modes, ${ }^{21}$ a viscous, non-modal instability mechanism that is sensitive to Reynolds number, with rapidly increasing amplification as Reynolds number increases. The eddy viscosity greatly attenuates these 


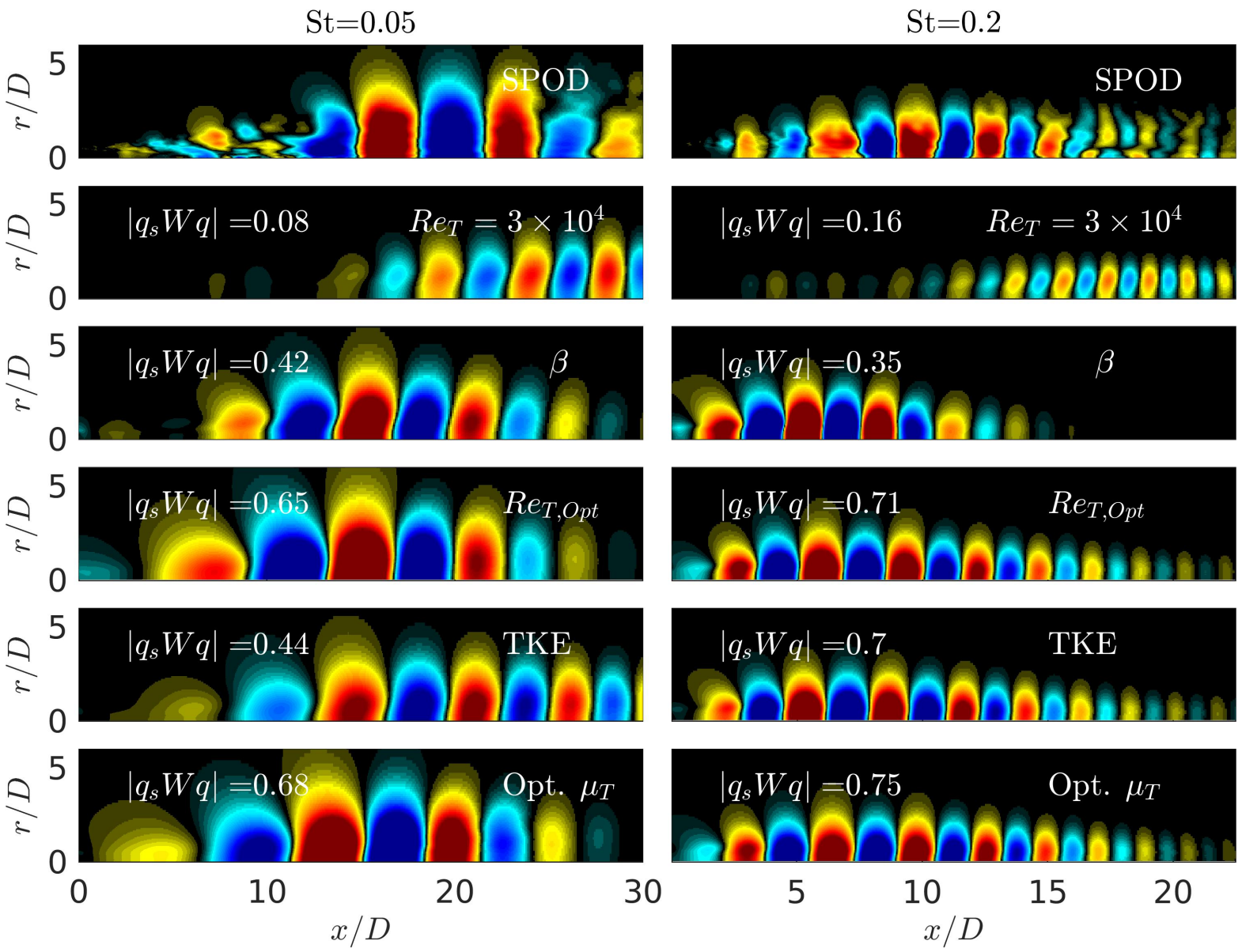

Figure 3. Real component of the response pressure fluctuations for $S t=0.05$ and $S t=0.2$ in the left and right columns respectively. Row 1 presents the optimal SPOD mode for which the optimization seeks to match. The following rows present results for $R e_{T}=3 \times 10^{4}$ and the optimal results for linear damping, constant eddy-viscosity field, turbulent kinetic energy model, and the full eddy-viscosity field optimization.

modes in favor of a Kelvin-Helmholtz (KH) response that peaks further upstream, consistent with what is observed in the SPOD.

Note that while we only plot the response pressure, we see similar trends and improvements in all flow variables similar to figure 2 .

Proceeding to higher frequencies, figure 4 displays the optimal fluctuating pressure modes for SPOD and the five resolvent methods for $S t=0.6$ and 1 . Projection coefficients are already quite high for these frequencies, as has been previously noted, ${ }^{21}$ but are further increased with the eddy-viscosity models, reaching an astonishing $96 \%$ for the optimal eddy viscosity. Here the differences in the mode shapes are subtle as the modes are generally shortened from the baseline case to better match the SPOD at both frequencies. Like the lower frequencies, the eddy-viscosity methods outperform the linear damping method as the eddy-viscosity terms address the Reynolds stresses.

At these higher frequencies, the jet response is a clear Kelvin-Helmholtz wavepacket, a modal, inviscid stability mechanism, and it is thus unsurprising that the results are relatively insensitive to the precise eddy-viscosity model. On the other hand, it is gratifying that the TKE and constant eddy-viscosity models perform almost as well as the optimized eddy-viscosity model.

For $S t=1$ the optimized projection coefficient are falling compared to the $S t=0.6$ case. This is again due to the emergence of Orr-modes with similar energy as the KH modes. By performing SPOD in limited domains near the nozzle exit, it becomes clear that the modal, low-rank $\mathrm{KH}$ response continues to much higher frequencies in the near nozzle region, ${ }^{45}$ but when considering the global response, these are always 


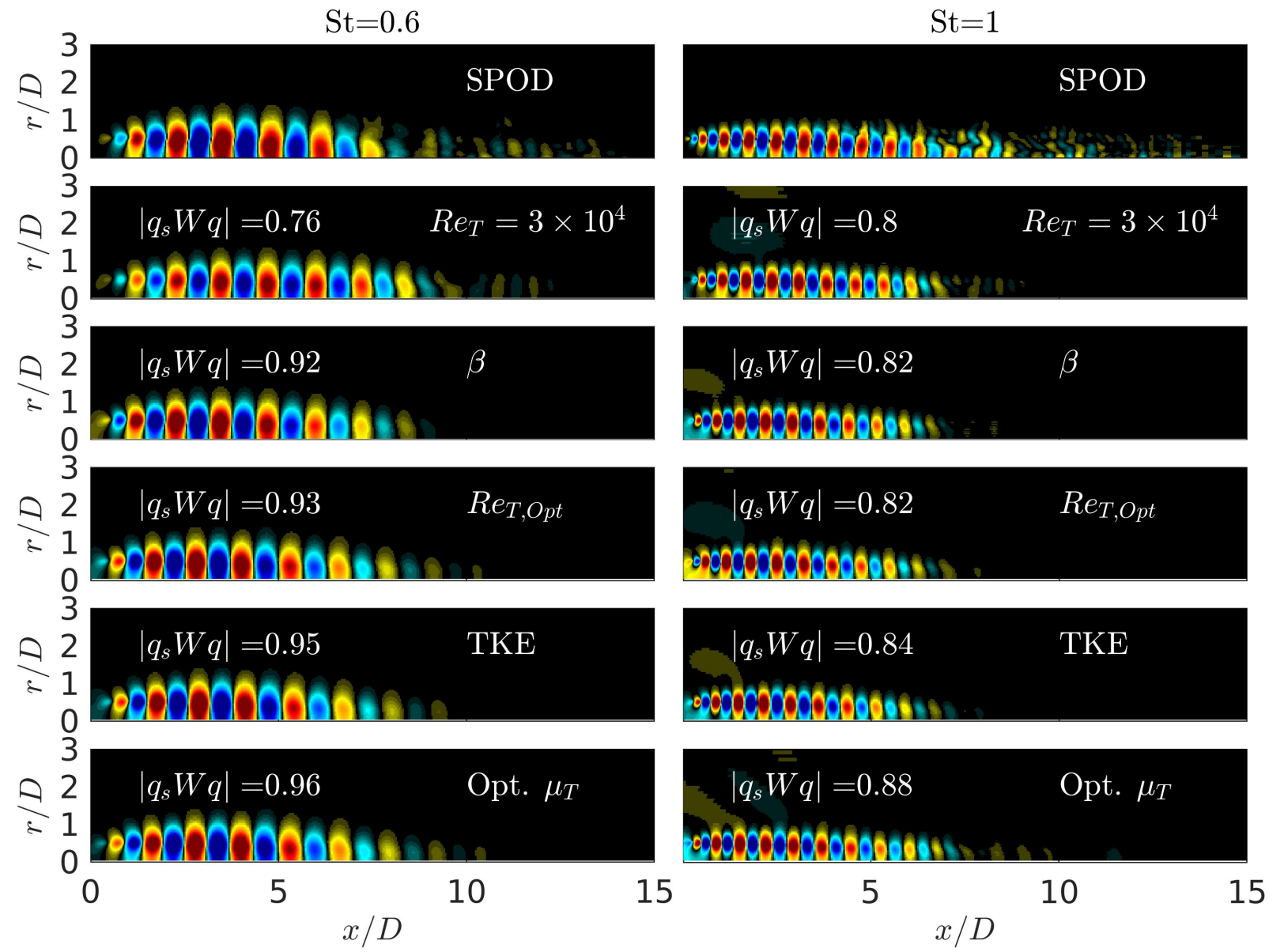

Figure 4. Real component of the response pressure fluctuations for $S t=0.6$ and $S t=1$ in the left and right columns respectively. Rows present the equivalent methods as described in figure 3 .

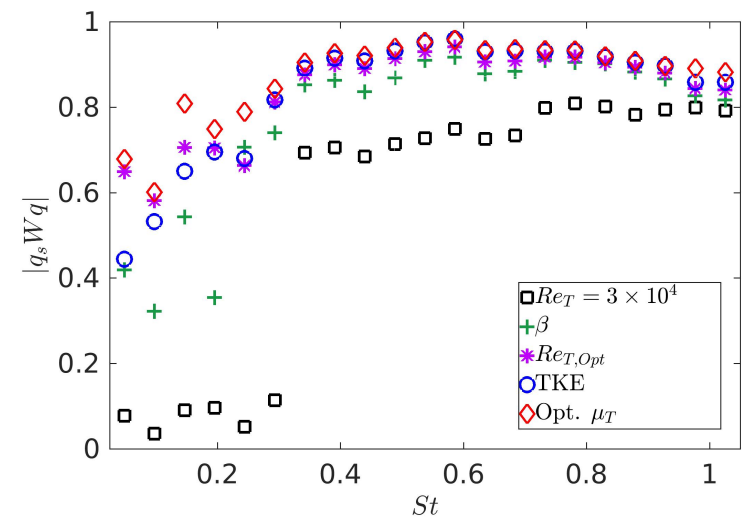

Figure 5. Optimal alignments for all methods investigated including $\operatorname{Re}_{T}=3 \times 10^{4}$.

inferior in energy to the Orr response being excited and peaking further downstream.

Performing the optimization across the frequency range $S t=0.05-1$ for all methods results in alignment 

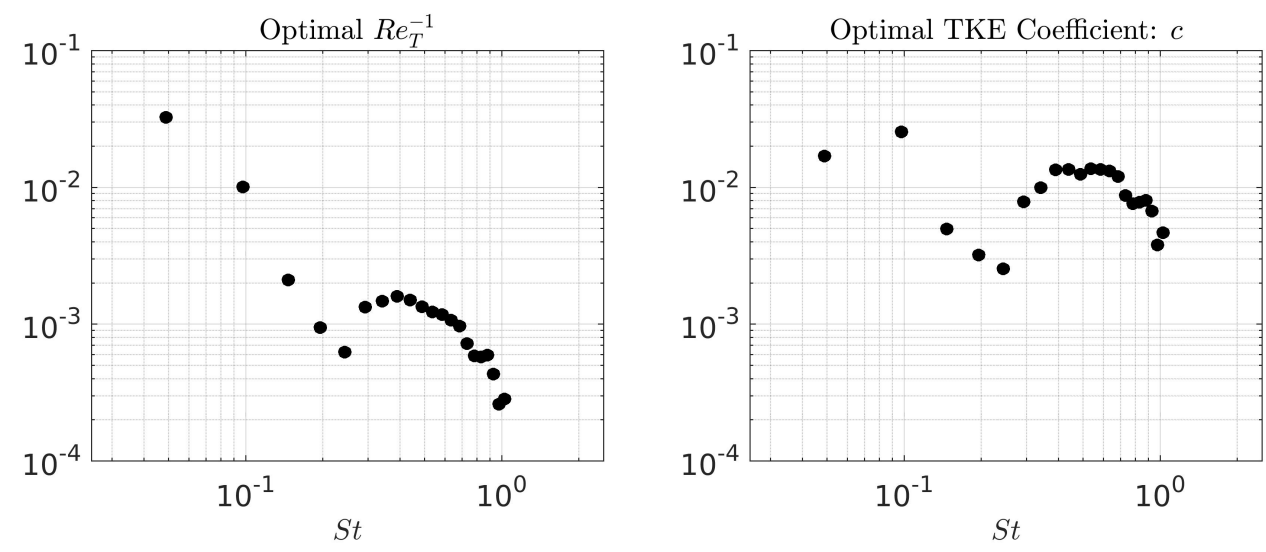

Figure 6. The optimal parameters across $S t=[0.05-1]$ for the optimal constant eddy-viscosity field (i.e. $R e_{T}$ ) and the optimal TKE eddy-viscosity model (i.e. c) in the left and right plots respectively.

coefficients displayed in figure 5. Throughout the frequency range considered, the alignments have generally improved considerably from the baseline case. Although we cannot be sure we found a global maximum, the optimal eddy-viscosity field provided the highest projection coefficients throughout the entire frequency range. However, the other eddy-viscosity models produce alignments that are quite close to the optimal eddyviscosity field. The constant eddy-viscosity is nearly optimal at lower frequencies (Orr-type modes), whereas the TKE model is more nearly optimal at higher ones. The linear damping model, while an improvement over the baseline case, is generally inferior to the eddy-viscosity models, and is not considered further.

\section{B. Optimal parameters}

We now turn our attention to the optimal parameters identified from optimal alignment of the eddy-viscosity models with SPOD.

The optimal parameters responsible for the increase in projection coefficients are shown in figure 6 for the constant eddy viscosity and TKE eddy-viscosity model. For the constant eddy-viscosity field there are three regions of interest from $S t=0.05-0.3, S t=0.3-0.8$ and $S t>0.8$. In the low frequency region, the baseline jet response consists of Orr-type modes that have a strong Reynolds number dependence, and relatively larger eddy viscosities are required to damp them. For $S t=0.05$ the ratio of molecular Reynolds number to effective Reynolds number is $R e_{j} / R e_{T} \approx 13,500$, impacting the resolvent mode by a four order of magnitude difference when compared to the molecular viscosity. The middle frequency regime is dominated by the inviscid $\mathrm{KH}$ mechanism and $R e_{j} / R e_{T} \approx 450$ is optimal, but recall from the optimization results above that the projection coefficients are high even without the eddy viscosity. In the higher frequency regime the response, in the baseline case, corresponds to a mix of $\mathrm{KH}$ and Orr-type waves, and the low-rank nature begins to diminish in the SPOD spectrum. As frequency increases the dependency on inverse effective Reynolds number begins to increase, similar to low frequencies, due to Orr-type modes and at the the highest frequency of our study, $S t=1$, we have $R e_{j} / R e_{T} \approx 135$.

Similar trends are observed for the optimal coefficient, $c$, for the TKE eddy-viscosity field plotted in figure 6. For both the low frequency and high frequency regions the coefficient is somewhat sensitive to frequency. However, over the KH dominated region the coefficient remains relatively constant. Although it is unclear why the scaling trends change as such, the end result is a TKE coefficient, $c$, which varies throughout $S t=0.05-1$ by only one order of magnitude.

For the full field eddy-viscosity optimization, we first recall that its primary purpose is to determine what may be an upper bound for how well any eddy-viscosity model could perform. Given that the alignments between the resolvent and SPOD modes were not significantly higher for the optimized scheme than for the modeled eddy-viscosity approaches (with optimal parameters), the detailed eddy-viscosity fields are of lesser importance. Still, some aspects of the physics are apparent in the optimized fields, which are presented in figure 7 for selected Strouhal numbers. From these fields there are three distinguishable forms the eddy- 

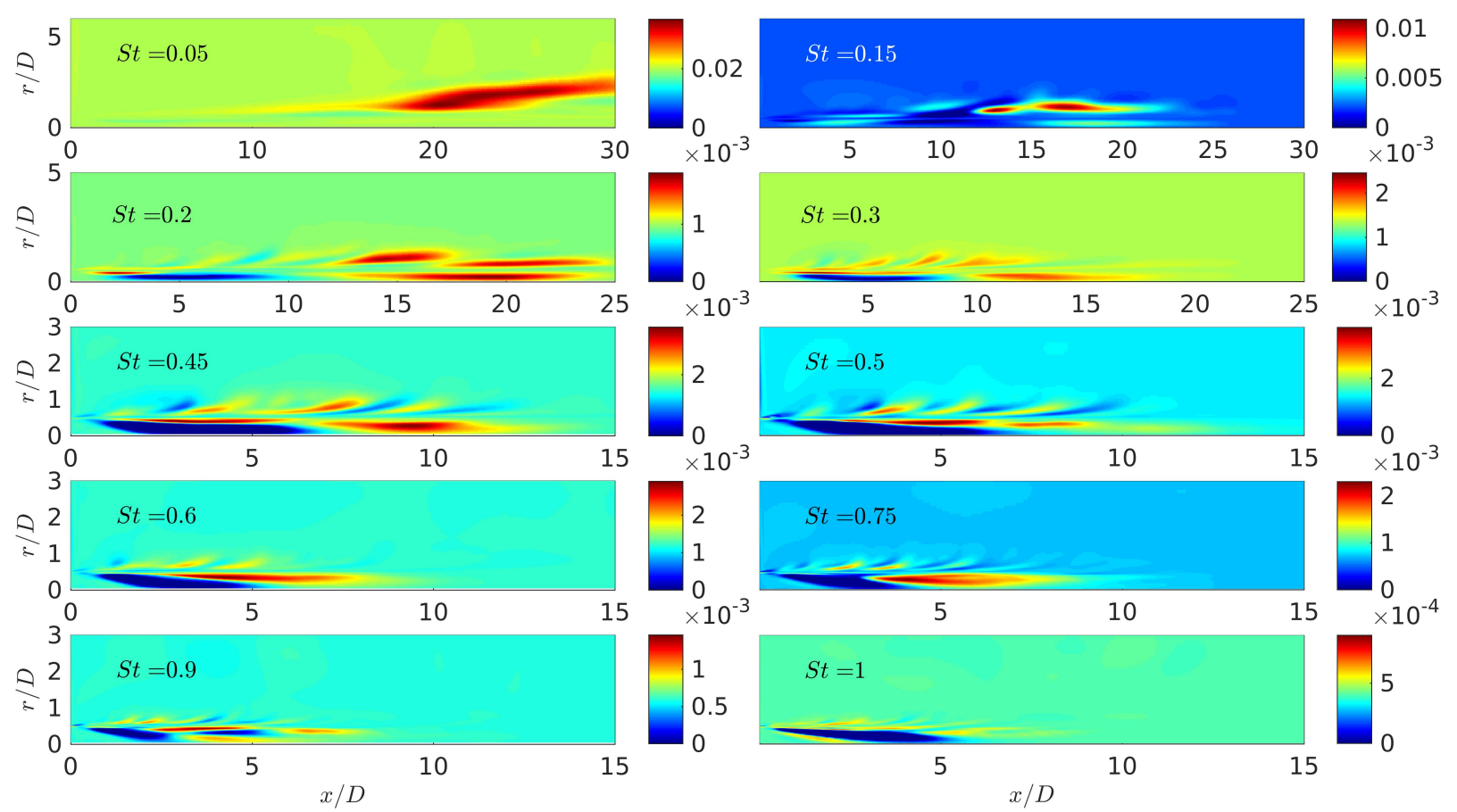

Figure 7. Optimal eddy-viscosity fields, $\mu_{T}$, for various Strouhal numbers from low frequency to high frequency proceeding left to right and top to bottom.

viscosity fields take. The first form is a downstream, off-centerline region of large eddy viscosity. In a sense, the addition of eddy viscosity represents a damping of the response mode and this can be understood by figure 3 where the resolvent mode computed via the optimal eddy-viscosity field is "pushed" upstream to match the observed SPOD mode. This behavior is seen up until $\sim S t=0.25$, for frequencies larger than $S t \geq 0.3$ the field changes character further upstream and resides along the potential core. This is associated with the change in optimal mechanisms from Orr to KH. Orr-type modes occur further downstream and off-centerline, while $\mathrm{KH}$ modes are active along the shear layer.

One interesting note from the full optimization eddy-viscosity fields are their spatial structure and general envelope. In sum these fields appear to construct an eddy-viscosity field similar to that of the TKE model. For low frequency fields, higher eddy viscosity is seen above $r / D=0.5$ and downstream relating to the portion of the TKE model above $r / D=0.5$ and moderate frequencies mirror the diagonal structure denoting the location of the shear layer also observed for $r / D<0.5$ in the TKE model. These insights begin to highlight support for a TKE eddy-viscosity model.

\section{Suboptimal modes}

The optimization currently only aligns the principle SPOD and resolvent modes. However, suboptimal modes are also of interest, particularly as they are known to be relevant for modeling coherence decay associated with 'jittering of wavepackets' to produce sound. ${ }^{46}$ Although the suboptimals could in theory also be better aligned through an optimal eddy viscosity, we are interested here in whether alignment with only the principle modes substantially alters the alignment of suboptimal modes, i.e. whether it diagonalizes the forcing CSD tensor. Using the optimal parameters for each method, the suboptimal modes are computed and shown in figure 8 for modes 2 and 3 and figure 9 for modes 4 and 5 .

Projection values are present for all comparisons and show significant improvement for the suboptimal modes when compared to the $R e_{T}=3 \times 10^{4}$ case which provides only $3 \%$ alignment for SPOD mode 2 . Not only are the mode shapes for the baseline case dissimilar, but the contour values of SPOD show the 
Mode 2

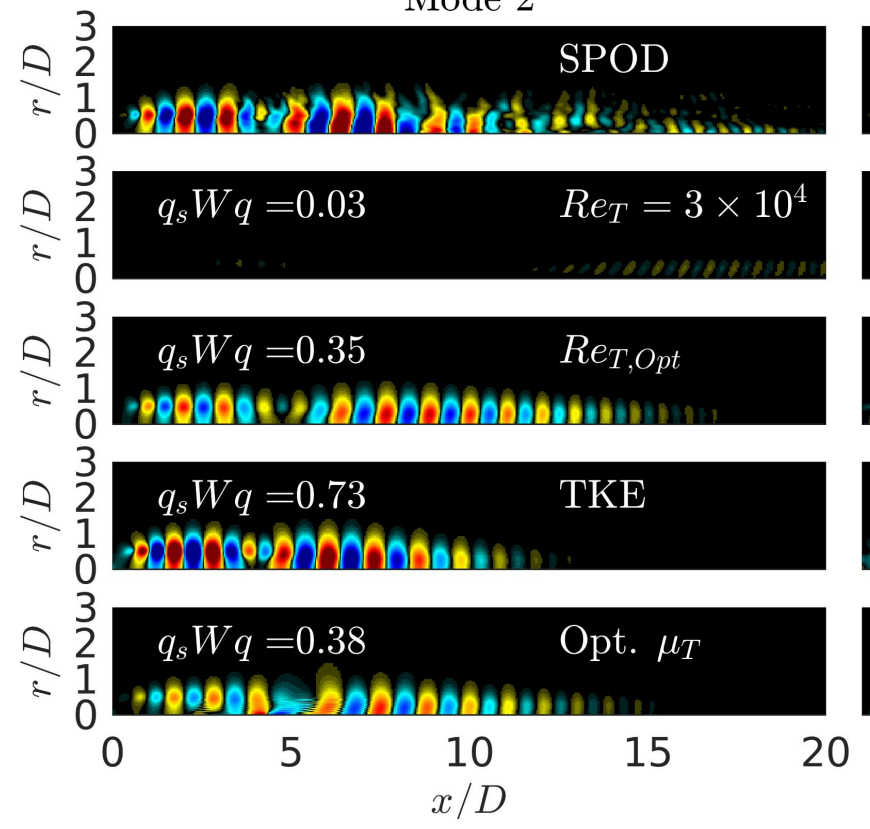

Mode 3
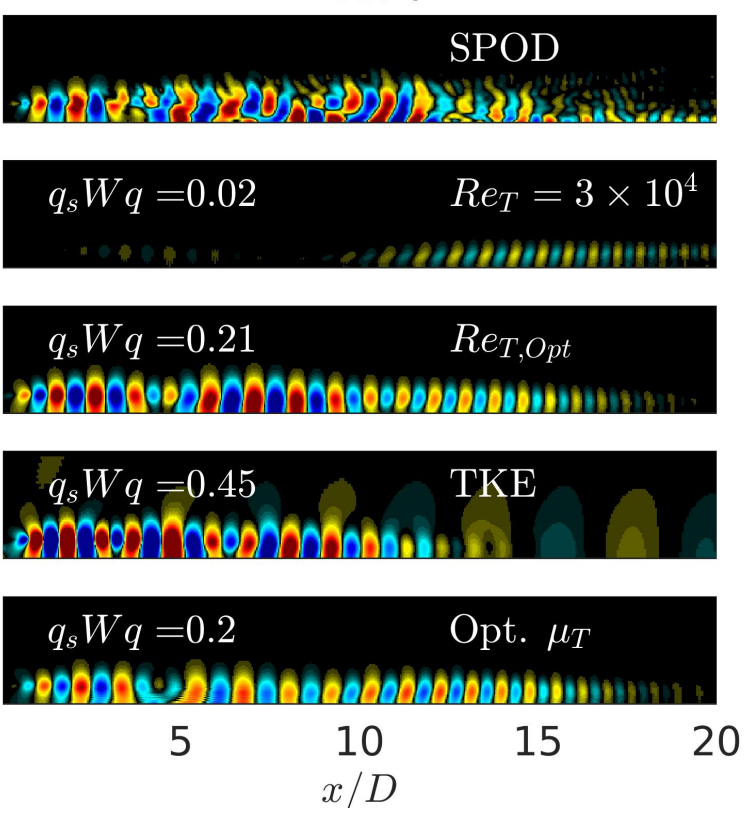

Figure 8. Suboptimal modes 2 and 3 at $S t=0.6$ in the left and right columns respectively for $\mathbf{S P O D}, R e_{T}=3 \times$ $10^{4}$, constant eddy-viscosity field, turbulent kinetic energy model, and the full eddy-viscosity field optimization.

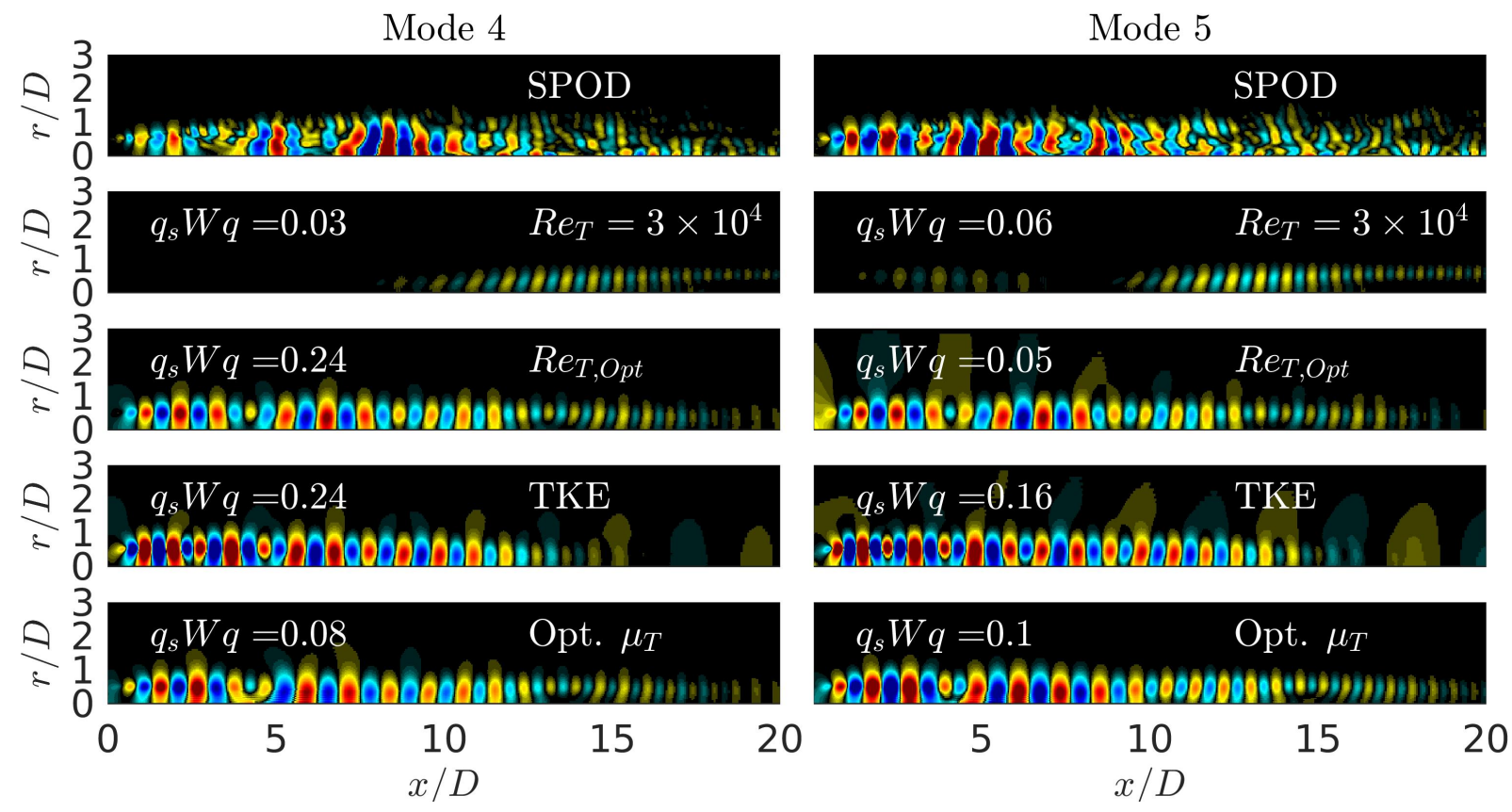

Figure 9. Suboptimal modes 4 and 5 at $S t=0.6$ in the left and right columns respectively for SPOD, $R e_{T}=3 \times$ $10^{4}$, constant eddy-viscosity field, turbulent kinetic energy model, and the full eddy-viscosity field optimization.

magnitudes of the $\operatorname{Re}_{T}=3 \times 10^{4}$ cases are drastically different, indicating that the distribution of energy between variables in this case is poor. In contrast, each of the eddy-viscosity models show increased alignment with the suboptimals. The greatest increase in alignment for suboptimal modes is via the optimized TKE 

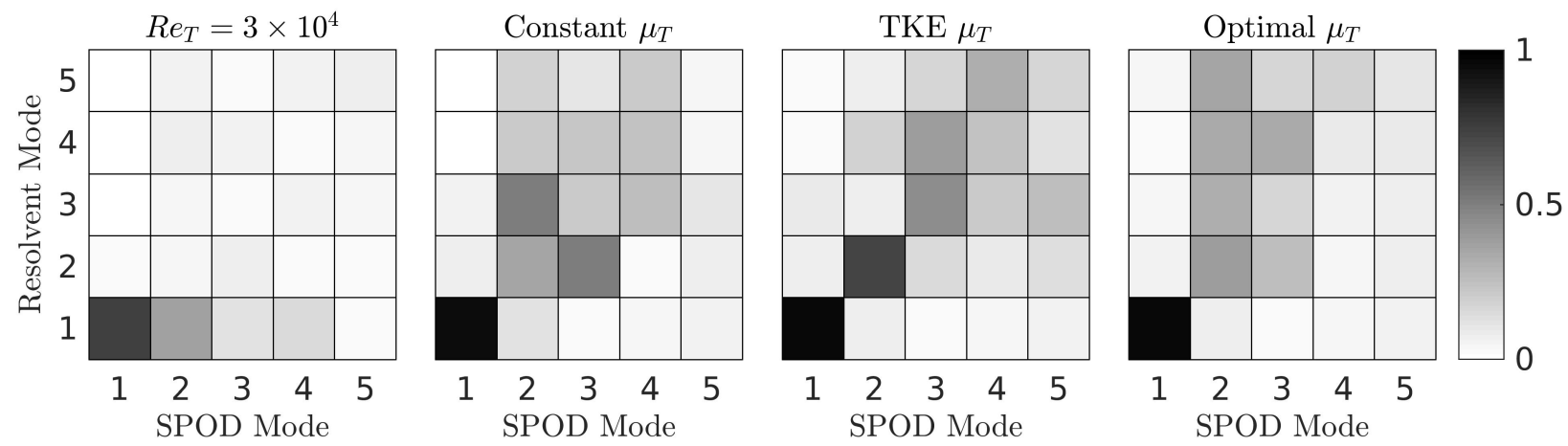

Figure 10. Projections of the first five SPOD modes into the first five resolvent modes determined for $R e_{T}=$ $3 \times 10^{4}$ and the three eddy-viscosity models at $S t=0.6$.

eddy-viscosity model, projecting to $73 \%$ for the first SPOD suboptimal and $45 \%$ with the second suboptimal. This observation provides significant support for the physical relevance of the TKE eddy-viscosity model. For suboptimal modes 4 and 5 in figure 9 the projection coefficients begin to rapidly deteriorate. This is attributable to the flattening of the SPOD spectrum and statistical limitations typically seen beyond the first few modes.

Assessing the suboptimals returns us to the question of colored-forcing in turbulent flows. As already discussed, the CSD tensor of the response may be related to CSD tensor of the forcing through the resolvent analysis. However, the CSD tensor for the forcing is generally unknown. In this study we instead assume the CSD tensor is white in space and attempt to adjust the resolvent operator such that white-noise forcing suffices. To assess how well our optimization of the first SPOD mode performed with regards to diagonalizing the forcing CSD, the projections of five SPOD modes are compared with the first five modes from each method in figure 10.

We first notice that the baseline case with $\operatorname{Re}_{T}=3 \times 10^{4}$ provides essentially no alignment of the suboptimal modes and thus does not diagonalize the coefficients. However, when considering each of the eddy-viscosity models we see both increased alignments with the suboptimals and a trend towards the diagonal. Here the TKE eddy-viscosity model is superior to even the optimal eddy viscosity, providing further evidence for physical relevance to the TKE eddy-viscosity model.

\section{Sensitivity of the TKE eddy-viscosity model}

Considering the good performance of the TKE model demonstrated in the previous sections, we turn our focus to how such a model should be tuned. As was shown in figure 6 the optimal TKE coefficient ranged from $c=0.02-0.004$, with a constant region at mid frequencies where $c=0.01$. However, the fully optimized eddy-viscosity field produced only marginally better alignment than the TKE model for most cases, and this suggests that the results may not be very sensitive to the precise constant. In this section, we sweep over frequencies but holding $c$ constant at values $c=[0.017,0.0135,0.01,0.0065]$. The resulting alignments are plotted versus frequency in figure 11.

All of the constants chosen provide acceptable alignments across the middle range of frequencies where the low-rank KH response is observed. Except for the outlier at $S t=0.1$, the constants $c=0.0065$ and 0.01 are suboptimal by only about $\sim 10-20 \%$. Thus overall we find that the TKE eddy-viscosity model is relatively insensitive to the scaling parameters. This suggests the structure of the TKE model is physically relevant and its implementation in further resolvent jet models is fruitful for prediction.

\section{Conclusions}

In this paper we test the extent to which eddy-viscosity models can be used to improve the alignment between the observed large-scale structures (deduced by SPOD) and the optimal resolvent mode. We formulated an optimization problem that allowed us to test different eddy-viscosity models and compare them to an optimal eddy-viscosity field that seeks the best alignment possible of any model. 


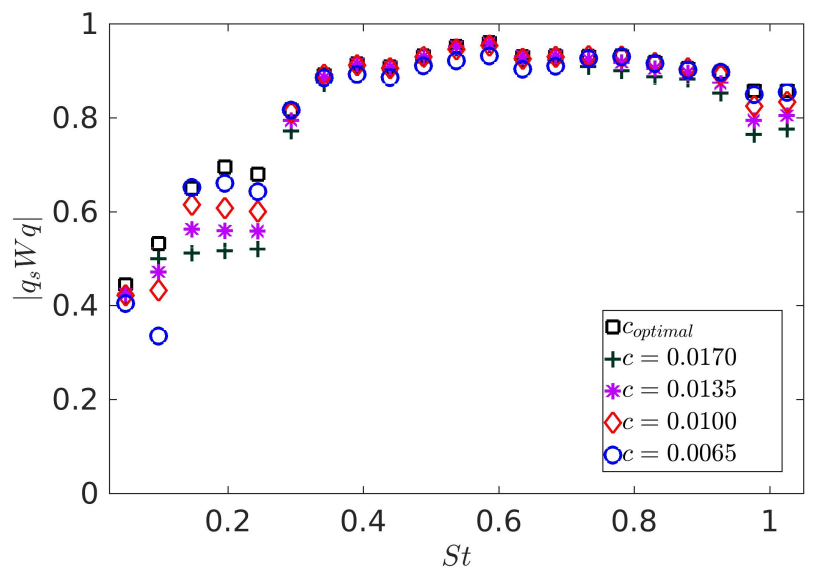

Figure 11. Alignments across all Strouhal numbers for various TKE eddy-viscosity model coefficients, $c=$ $[1.7,1.35,1,0.65] \times 10^{-2}$, and compared with the optimal TKE coefficient at each frequency.

Regardless of the specific model, we obtained substantial improvements in alignment compared to a baseline case that used a constant eddy viscosity corresponding to a value of $R e_{T}=30,000$. Across the frequencies considered, the addition of eddy viscosity served to highlight the role different mechanisms at play for the $m=0$ mode we considered, namely, Orr-type and KH-type instabilities. The Orr-type mechanism is sensitive to the eddy-viscosity fields, whether at low or high frequencies as the optimal mode or in the suboptimal modes. The KH-type mode however is relatively insensitive to the eddy-viscosity field, a result expected from the inviscid nature of the inflectional $\mathrm{KH}$ instability.

Suboptimal modes were also assessed for the optimal parameters and significant improvements were found. All eddy-viscosity models emitted suboptimal modes that were more closely aligned with suboptimal SPOD modes than in the baseline case. In particular, the TKE model presented the greatest diagonalization of the forcing CSD, providing evidence for the model's physical relevance.

To assess the sensitivity to the optimal TKE coefficients, four constant values were chosen and their ability to model the SPOD modes throughout the frequency range was shown. All constant coefficients provided reasonable agreement across the entire frequency range, giving only marginal losses in projections when compared to the optimal TKE coefficient projections. Two constants of superior performance were $c=0.0065,0.01$. Considering the relative simplicity and insensitivity of the TKE model, we suggest its implementation in future resolvent jet models for increased model accuracy.

Future work looks to better assess the role of SPOD statistical errors on the optimization and extensions of the analysis to the remainder of the parameter space in jets, $m>0$, various Mach numbers, and towards an optimization specifically designed for far-field noise. Additionally, we wish to investigate and assess the applicability of modern eddy-viscosity models used throughout the literature.

\section{Acknowledgments}

This research was supported by a grant from the Office of Naval Research (grant No. N00014-16-1-2445) with Dr. Steven Martens as program manager. E.P. was supported by the Department of Defense (DoD) through the National Defense Science Engineering Graduate Fellowship (NDSEG) Program. The LES study was performed at Cascade Technologies, with support from ONR and NAVAIR SBIR project, under the supervision of Dr. John T. Spyropoulos. The main LES calculations were carried out on DoD HPC systems in ERDC DSRC.

\section{References}

${ }^{1}$ Lighthill, M. J., "On sound generated aerodynamically I. General theory," Proc. R. Soc. Lond. A, Vol. 211, No. 1107, 1952 , pp. $564-587$. 
${ }^{2}$ Goldstein, M. E., “A generalized acoustic analogy," Journal of Fluid Mechanics, Vol. 488, 2003, pp. 315-333.

${ }^{3}$ Mollo-Christensen, E., "Jet noise and shear flow instability seen from an experimenters viewpoint," Journal of Applied Mechanics, Vol. 34, No. 1, 1967, pp. 1-7.

"Jordan, P. and Colonius, T., "Wave packets and turbulent jet noise," Annual Review of Fluid Mechanics, Vol. 45, 2013, pp. $173-195$.

${ }^{5}$ Papamoschou, D., "Wavepacket modeling of the jet noise source," International Journal of Aeroacoustics, Vol. 17, No. 1-2, 2018, pp. 52-69.

${ }^{6}$ Jeun, J. and Nichols, J. W., "Input-output analysis of Mach 0.9 jet noise," arXiv preprint arXiv:1806.09280, 2018.

${ }^{7}$ Michalke, A., "Instability of a compressible circular free jet with consideration of the influence of the jet boundary layer thickness," Z. für Flugwissenschaften, Vol. 19, No. 8, 1971, pp. 319-328.

${ }^{8}$ Crighton, D. G. and Gaster, M., "Stability of slowly diverging jet flow," Journal of Fluid Mechanics, Vol. 77, No. 02, 1976, pp. 397-413.

${ }^{9}$ Gudmundsson, K. and Colonius, T., "Instability wave models for the near-field fluctuations of turbulent jets," Journal of Fluid Mechanics, Vol. 689, 2011, pp. 97-128.

${ }^{10}$ Nichols, J. W. and Lele, S. K., "Global modes and transient response of a cold supersonic jet," Journal of Fluid Mechanics, Vol. 669, 2011, pp. 225-241.

${ }^{11}$ Goldstein, M. and Leib, S., "The aeroacoustics of slowly diverging supersonic jets," Journal of Fluid Mechanics, Vol. 600, 2008, pp. 291-337.

${ }^{12}$ Goldstein, M. E. and Leib, S. J., "Azimuthal Source Non-Compactness and Mode Coupling in Sound Radiation from High-Speed Axisymmetric Jets," 22nd AIAA/CEAS Aeroacoustics Conference, 2016, p. 2803.

${ }^{13}$ Gryazev, V. and Karabasov, S. A., "Comparison of two Goldstein acoustic analogy implementations with the Tam\&Auriault model for heated and unheated jet noise prediction," 2018 AIAA/CEAS Aeroacoustics Conference, 2018, p. 2828.

${ }^{14}$ Semiletov, V. A. and Karabasov, S. A., "A volume integral implementation of the Goldstein generalised acoustic analogy for unsteady flow simulations," Journal of Fluid Mechanics, Vol. 853, 2018, pp. 461-487.

${ }^{15}$ Papamoschou, D., "Modelling of noise reduction in complex multistream jets," Journal of Fluid Mechanics, Vol. 834, 2018 , pp. 555-599.

${ }^{16} \mathrm{McKeon}, \mathrm{B}$. and Sharma, A., "A critical-layer framework for turbulent pipe flow," Journal of Fluid Mechanics, Vol. 658, 2010, pp. 336-382.

${ }^{17}$ Jovanović, M. R. and Bamieh, B., "Componentwise energy amplification in channel flows," Journal of Fluid Mechanics, Vol. 534, 2005, pp. 145-183.

${ }^{18}$ Jeun, J., Nichols, J. W., and Jovanović, M. R., "Input-output analysis of high-speed axisymmetric isothermal jet noise," Physics of Fluids, Vol. 28, No. 4, 2016, pp. 047101.

${ }^{19}$ Nichols, J. W. and Jovanovic, M. R., "Input-output analysis of high-speed jet noise," Proceedings of the Summer Program, Center for Turbulence Research, Stanford University, 2014.

${ }^{20}$ Schmidt, O. T., Colonius, T., and Bres, G. A., "Linear dynamics of large-scale structures in turbulent jets," Tenth International Symposium on Turbulence and Shear Flow Phenomena, 2017.

${ }^{21}$ Schmidt, O. T., Towne, A., Rigas, G., Colonius, T., and Brès, G. A., "Spectral analysis of jet turbulence," Journal of Fluid Mechanics, Vol. 855, 2018, pp. 953-982.

${ }^{22}$ Rigas, G., Schmidt, O. T., Colonius, T., and Bres, G. A., "One-Way Navier-Stokes and resolvent analysis for modeling coherent structures in a supersonic turbulent jet," 23rd AIAA/CEAS Aeroacoustics Conference, Vol. 4046, 2017.

${ }^{23}$ Towne, A., Schmidt, O. T., and Colonius, T., "Spectral proper orthogonal decomposition and its relationship to dynamic mode decomposition and resolvent analysis," Journal of Fluid Mechanics, Vol. 847, 2018, pp. 821-867.

${ }^{24}$ Brès, G. A., Jordan, P., Colonius, T., Le Rallic, M., Jaunet, V., and Lele, S. K., "Large eddy simulation of a Mach 0.9 turbulent jet," Proceedings of the Summer Program, Center for Turbulence Research, Stanford University, 2014.

${ }^{25}$ Brès, G. A., Jaunet, V., Le Rallic, M., Jordan, P., Colonius, T., and Lele, S. K., "Large eddy simulation for jet noise: the importance of getting the boundary layer right," AIAA paper 2015-2535, 2015.

${ }^{26}$ Zare, A., Jovanovi, M. R., and Georgiou, T. T., "Colour of turbulence," Journal of Fluid Mechanics, Vol. 812, 2017, pp. 636680 .

${ }^{27}$ Towne, A., "Completing partially known space-time flow statistics: A resolvent-based approach," .

${ }^{28}$ Crouch, J., Garbaruk, A., and Magidov, D., "Predicting the onset of flow unsteadiness based on global instability," Journal of Computational Physics, Vol. 224, No. 2, 2007, pp. 924-940.

${ }^{29}$ Oberleithner, K., Paschereit, C. O., and Wygnanski, I., "On the impact of swirl on the growth of coherent structures," Journal of Fluid Mechanics, Vol. 741, 2014, pp. 156-199.

${ }^{30}$ Rukes, L., Paschereit, C. O., and Oberleithner, K., "An assessment of turbulence models for linear hydrodynamic stability analysis of strongly swirling jets," European Journal of Mechanics-B/Fluids, Vol. 59, 2016, pp. 205-218.

${ }^{31}$ Sartor, F., Mettot, C., and Sipp, D., "Stability, receptivity, and sensitivity analyses of buffeting transonic flow over a profile," AIAA Journal, Vol. 53, No. 7, 2014, pp. 1980-1993.

${ }^{32}$ Tammisola, O. and Juniper, M. P., "Coherent structures in a swirl injector at $\mathrm{Re}=4800$ by nonlinear simulations and linear global modes," Journal of Fluid Mechanics, Vol. 792, 2016, pp. 620-657.

${ }^{33}$ Mettot, C., Sipp, D., and Bézard, H., "Quasi-laminar stability and sensitivity analyses for turbulent flows: prediction of low-frequency unsteadiness and passive control," Physics of Fluids, Vol. 26, No. 4, 2014, pp. 061701.

${ }^{34}$ Morra, P., Semeraro, O., Henningson, D. S., and Cossu, C., "On the relevance of Reynolds stresses in resolvent analyses of turbulent wall-bounded flows," arXiv preprint arXiv:1901.04356, 2019.

${ }^{35}$ Mattsson, K. and Nordström, J., "Summation by parts operators for finite difference approximations of second derivatives," Journal of Computational Physics, Vol. 199, No. 2, 2004, pp. 503-540. 
${ }^{36}$ Mohseni, K. and Colonius, T., "Numerical treatment of polar coordinate singularities," Journal of Computational Physics, Vol. 157, No. 2, 2000, pp. 787-795.

${ }^{37} \mathrm{Chu}$, B.-T., "On the energy transfer to small disturbances in fluid flow (Part I)," Acta Mechanica, Vol. 1, No. 3, 1965, pp. 215-234.

${ }^{38}$ Lumley, J. L., "The structure of inhomogeneous turbulent flows," Atmospheric turbulence and radio propagation, 1967, pp. $166-178$.

${ }^{39}$ Lumley, J. L., Stochastic tools in turbulence, New York: Academic Press, 1970.

${ }^{40}$ Semeraro, O., Jaunet, V., Jordan, P., Cavalieri, A. V., and Lesshafft, L., "Stochastic and harmonic optimal forcing in subsonic jets," 22nd AIAA/CEAS Aeroacoustics Conference, 2016, p. 2935.

${ }^{41}$ Towne, A., Bres, G. A., and Lele, S. K., "A statistical jet-noise model based on the resolvent framework," 23rd AIAA/CEAS Aeroacoustics Conference, 2017, p. 3706.

${ }^{42}$ Jovanović, M. R., Modeling, analysis, and control of spatially distributed systems, University of California at Santa Barbara, Dept. of Mechanical Engineering, 2004.

${ }^{43}$ Yeh, C.-A. and Taira, K., "Resolvent-analysis-based design of airfoil separation control," Journal of Fluid Mechanics, Vol. 867, 2019, pp. 572-610.

${ }^{44}$ Pope, S. B., "Turbulent flows," 2001.

${ }^{45}$ Sasaki, K., Cavalieri, A. V., Jordan, P., Schmidt, O. T., Colonius, T., and Brès, G. A., "High-frequency wavepackets in turbulent jets," Journal of Fluid Mechanics, Vol. 830, 2017.

${ }^{46}$ Cavalieri, A. V., Jordan, P., Agarwal, A., and Gervais, Y., "Jittering wave-packet models for subsonic jet noise," Journal of Sound and Vibration, Vol. 330, No. 18-19, 2011, pp. 4474-4492. 